\title{
O gênero Lemnicola (Bacillariophyceae) no Estado de São Paulo, Brasil
}

\author{
Luisiana Andresa Carneiro ${ }^{1}$ e Denise de Campos Bicudo ${ }^{1,2}$
}

Recebido: 28.06.2006; aceito: 11.06.2007

\begin{abstract}
Genus Lemnicola (Bacillariophyceae) in the State of São Paulo, Brazil). The study aimed at contributing to the diatom biodiversity of the State of São Paulo, southeastern Brazil, particularly to genus Lemnicola. Ninety-six samples were collected, representing 64 municipalities of the state, including plankton and periphyton of lentic and lotic systems. Lemnicola hungarica (Grunow) Round \& Basson, the genus single species, was always found associated to submerged substrata. It was registered in three municipalities (Itapura, São Paulo and Reginópolis), indicating the restrict distribution of the genus in the state. Genus is cited for the first time for São Paulo State, and ultrastructure (SEM) information is pioneer for Brazilian specimens.
\end{abstract}

Key words: Achnanthidiaceae, diatoms, taxonomy

RESUMO - (O gênero Lemnicola (Bacillariophyceae) no Estado de São Paulo, Brasil). O trabalho visa contribuir com o conhecimento da biodiversidade de diatomáceas no Estado de São Paulo e, particularmente, do gênero Lemnicola. Foram examinadas 96 amostras coletadas em 64 municípios distribuídos no Estado de São Paulo, incluindo materiais planctônicos e perifíticos de ambientes lóticos e lênticos. A única espécie do gênero, L. hungarica (Grunow) Round \& Basson, foi documentada invariavelmente associada a superfícies submersas. Distribuiu-se em três municípios (Itapura, São Paulo e Reginópolis), indicando ter pequena distribuição no estado. O gênero é citado pioneiramente para o Estado de São Paulo, bem como são inéditas as descrições e ilustrações de características ultra-estruturais (MEV) para material brasileiro.

Palavras-chave: Achnanthidiaceae, diatomáceas, taxonomia

\section{Introdução}

Os trabalhos sobre diatomáceas ainda são escassos na América Tropical apesar do interesse que se tem pela grande diversidade biológica dessa região (Sala et al. 1999).

Principalmente a partir da década de 90 do século passado, várias mudanças ocorreram em nível de gênero e família, visando acomodar a descoberta e inclusão de novas características ultra-estruturais das frústulas dos representantes de diatomáceas (Round et al. 1990). Particularmente a ordem Achnanthales passou a ser dividida em três famílias, sendo que Achnanthidiaceae abriga, no presente, oito gêneros, dentre os quais Lemnicola, que são primordialmente distribuídos em águas dulciaqüícolas. Ressalta-se que as contribuições de Bukhtiyarova \& Round (1996), Round \& Bukhtiyarova (1996a) e Round \& Basson (1997) foram substanciais na composição genérica de Achnanthidiaceae.

A partir da revisão de literatura em âmbito sulamericano, observou-se que Lemnicola foi registrado em apenas três países (Colômbia, Argentina e Brasil), sendo que a maior parte das citações faz parte de listagens taxonômicas, com poucos registros que incluam ilustrações dos materiais examinados. A primeira citação de Lemnicola hungarica (Grun.) Round \& Basson (como Achnanthes hungarica Grunow) para América do Sul foi feita por West (1914), com base em estudo realizado em uma província colombiana andina. Quase 30 anos mais tarde, Frenguelli (1942) menciona o táxon pela segunda vez a partir de material da Patagônia, mais especificamente na Província de Neuquén, Argentina. Ainda para este país, existem estudos mais recentes, como os de Herbst \& Maidana (1989) e Maidana \& Herbst (1994) para amostras provenientes de vários corpos d'água na província de Chaco; De Domitrovic \& Maidana (1997) para o Rio Paraná, bem como Maidana et al. (1998) para a lagoa de Pozuelos (Jujuy). Finalmente, a espécie também foi reportada na Argentina para o lago Pulmarí, localizado na província de Neuquén por Diaz-Villanueva \& Maidana (1999) e Maidana \& Diaz-Villanueva (2001). Todas

1. Instituto de Botânica, Caixa Postal 3005, 01061-970 São Paulo, SP, Brasil

2. Autor para correspondência: dbicudo@terra.com.br 
as publicações acima se referem à espécie como Achnanthes hungarica Grunow em listagens taxonômicas, sem qualquer ilustração. Até o momento, com base em material da Amazônia Colombiana, Sala et al. (1999) consiste na única publicação de cunho efetivo para América do Sul que descreve e ilustra o táxon em microscopia eletrônica de varredura (MEV), porém somente parte da valva rafídea.

Particularmente no Brasil, os estudos diatomológicos em ambientes dulciaqüícolas estão concentrados nas regiões Sul, Sudeste e no Distrito Federal, nada ou pouco se conhecendo para as demais regiões. A primeira citação de Lemnicola hungarica (Grun.) Round \& Basson (como Achnanthes hungarica Grunow) no país encontra-se em Aguiar \& Martau (1979), a partir de amostras provenientes do Parque Zoológico de Porto Alegre, Estado do Rio Grande do Sul. Com base no levantamento de táxons listados para este estado, Torgan et al. (1999) mencionam duas citações contidas em Rosa et al. (1987) e Flores (1997), para a região de Charqueadas e do Banhado do Taim, respectivamente. No Estado do Paraná existem referências acompanhadas de ilustrações. Assim, para o Município de Curitiba, Contin $(1983,1990)$ registra a espécie para uma área de captação de água do Rio Iguaçu, Shirata (1986) para o lago do Parque São Lourenço e Visinoni (2002) para rios da região metropolitana. Ainda para este Estado, Valente-Moreira et al. (1994) menciona para Paranaguá, Leandrini (1999) para Maringá, Ludwig \& Flôres (1995) em Mangueirinha, para a região da Usina Hidrelétrica de Segredo, Brassac (1999) para sistemas lóticos da área de influência da Usina Hidrelétrica de Salto Caxias (Bacia do Rio Iguaçu) e Atab (2000), para rios das Bacias do Rio Tibagi e Itararé. Finalmente, Ferrari (2004) menciona para o Município de Prudentópolis e Landucci (2002) e Bigunas (2005) para rios situados em regiões litorâneas. Para o Estado de Santa Catarina foram registrados os trabalhos de Rodrigues (1984), Rodrigues (1988) e Rodrigues \& Moreira Filho (1990) que citaram e ilustraram o táxon para o Rio Tubarão. Finalmente, para o Estado do Rio de Janeiro, foi registrada uma citação ilustrada em Costa (1995) para a Reserva Biológica de Poço das Antas, no Município de Silva Jardim.

Este trabalho insere-se em contexto mais amplo e visa contribuir com o conhecimento da biodiversidade de diatomáceas no Estado de São Paulo. Contribui, pioneiramente, com o conhecimento florístico do gênero Lemnicola, bem como fornece descrições completas, incluindo ilustrações de características ultra-estruturais (MEV), inéditas para material brasileiro.

\section{Material e métodos}

Foram examinadas 96 amostras coletadas em 64 municípios distribuídos de forma mais uniforme possível no Estado de São Paulo (figura 1). As amostragens incluíram materiais planctônicos e perifíticos (associados a plantas, rochas e sedimentos) de ambientes lóticos e lênticos. O material planctônico foi coletado com rede de náilon (abertura de $20 \mu \mathrm{m}$ ) e o perifítico, mediante espremido de plantas aquáticas submersas, raspagem de substratos submersos ou, ainda, da retirada de amostras da camada superficial de sedimento na zona litorânea. As amostras foram imediatamente fixadas em campo com formalina a 3-5\%. A oxidação do material e o preparo das lâminas permanentes seguiram Hasle \& Fryxel (1970) e Simonsen (1974), modificado por Moreira Filho \& Valente-Moreira (1981), utilizando Hyrax como meio de inclusão.

As amostras analisadas, bem como as lâminas permanentes confeccionadas, encontram-se depositadas no acervo do Herbário Científico do Estado "Maria Eneida P. Kauffmann Fidalgo" (SP) do Instituto de Botânica da Secretaria do Meio Ambiente do Estado de São Paulo. Apenas as amostras com o registro da espécie foram incluídas em "materiais examinados". A terminologia adotada seguiu Anonymous (1975), Ross et al. (1979), Barber \& Haworth (1981) e Round et al. (1990). O sistema de classificação adotado foi o de Round et al. (1990), incluindo a proposição de Round \& Basson (1997). Finalmente, as ilustrações foram feitas a partir de microscópio fotônico Carl Zeiss modelo Axioskop 2 e microscópio eletrônico de varredura Jeol modelo LSM-840A a $25 \mathrm{kv}$.

\section{Resultados e Discussão}

O gênero monoespecífico Lemnicola foi proposto por Round \& Basson (1997) para incluir Achnanthes hungarica Grunow. Os autores afirmaram que aparentemente $A$. hungarica apresenta certas características semelhantes a Planothidium Round \& Bukhtiyarova. Todavia concordaram com Krammer \& Lange-Bertalot (1991) em separar este táxon do grupo "lanceolata", formado pelo gênero 


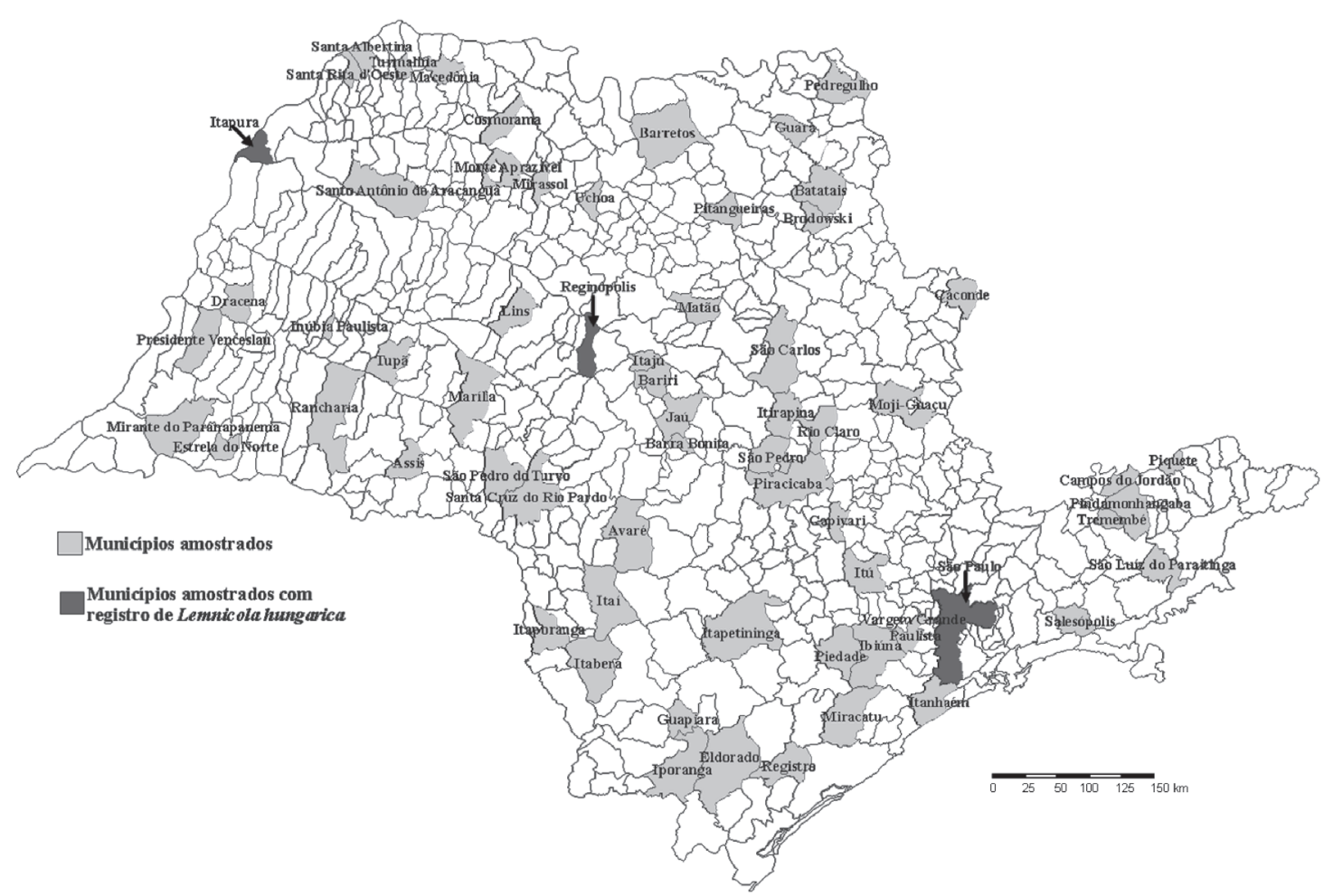

Figura 1. Municípios no Estado de São Paulo nos quais foram coletadas amostras e, em destaque (cinza), onde ocorreram representantes de Lemnicola.

Planothidium. Esta separação fundamentou-se principalmente em três características: na forma do estauro central na valva rafídea (área central bilateralmente expandida de modo simétrico e assimétrico, respectivamente em Planothidium e Lemnicola), nas estrias (tri- ou multisseriadas em Planothidium e bisseriadas em Lemnicola) e no formato externo da extremidade distal da rafe (curvadas para a mesma direção em Planothidium e para direção oposta em Lemnicola). Os autores mencionaram, ainda, a ausência de manto areolado como outra característica distinguível de Planothidium. Entretanto, estudos ao MEV (Round et al. 1990, Round \& Bukhtiyarova 1996b, Carneiro 2003) demonstram que Planothidium também apresenta esta característica.

Lemnicola hungarica (Grunow) Round \& Basson, Diatom Reseach 12: 71-81. 1997. Achnanthidium hungaricum Grunow in: Verhandlungen der kaiserlich-königlichen Zoologisch-Botanischen Gesellschaft in Wien, 13:146. 1863.

Figuras 2-9

Valvas lanceoladas a linear-lanceoladas; extremidades cuneadas, sub-rostradas ou rostradas; estrias transapicais paralelas na porção mediana da valva e radiadas em direção à porção distal; areolação inconspícua em microscopia fotônica. Valva rafídea: esterno linear estreito, com extremidades curvadas em direções opostas; área central transversalmente expandida, bilateralmente de forma assimétrica (trapeziforme de um lado e retangular de outro), alcançando as margens valvares; rafe reta na região proximal, curvada distalmente em direções opostas; estrias paralelas e radiadas em direção às extremidades, ausentes ou encurtadas na região central. Valva arrafídea: esterno linear estreito, extremidades retas; área central ausente, irregular formada pelo encurtamento das estrias centrais ou reduzida de formato circular; estrias de tamanho igual ao longo da valva ou encurtadas na região central formando uma área central circular ou irregular. MEV - Valva arrafídea, face interna: Interestrias fortemente espessas delimitando agrupamentos de estrias bisseriadas, manto raso. Medidas - eixo apical: 18,5-29,5 $\mu \mathrm{m}$; eixo transapical: 6-8,1 $\mu \mathrm{m}$; RC/L (razão entre comprimento e largura): 2,9-4,3; valva rafídea: 17-22 estrias em $10 \mu \mathrm{m}$; valva arrafídea: 15-21 estrias em $10 \mu \mathrm{m}$. 

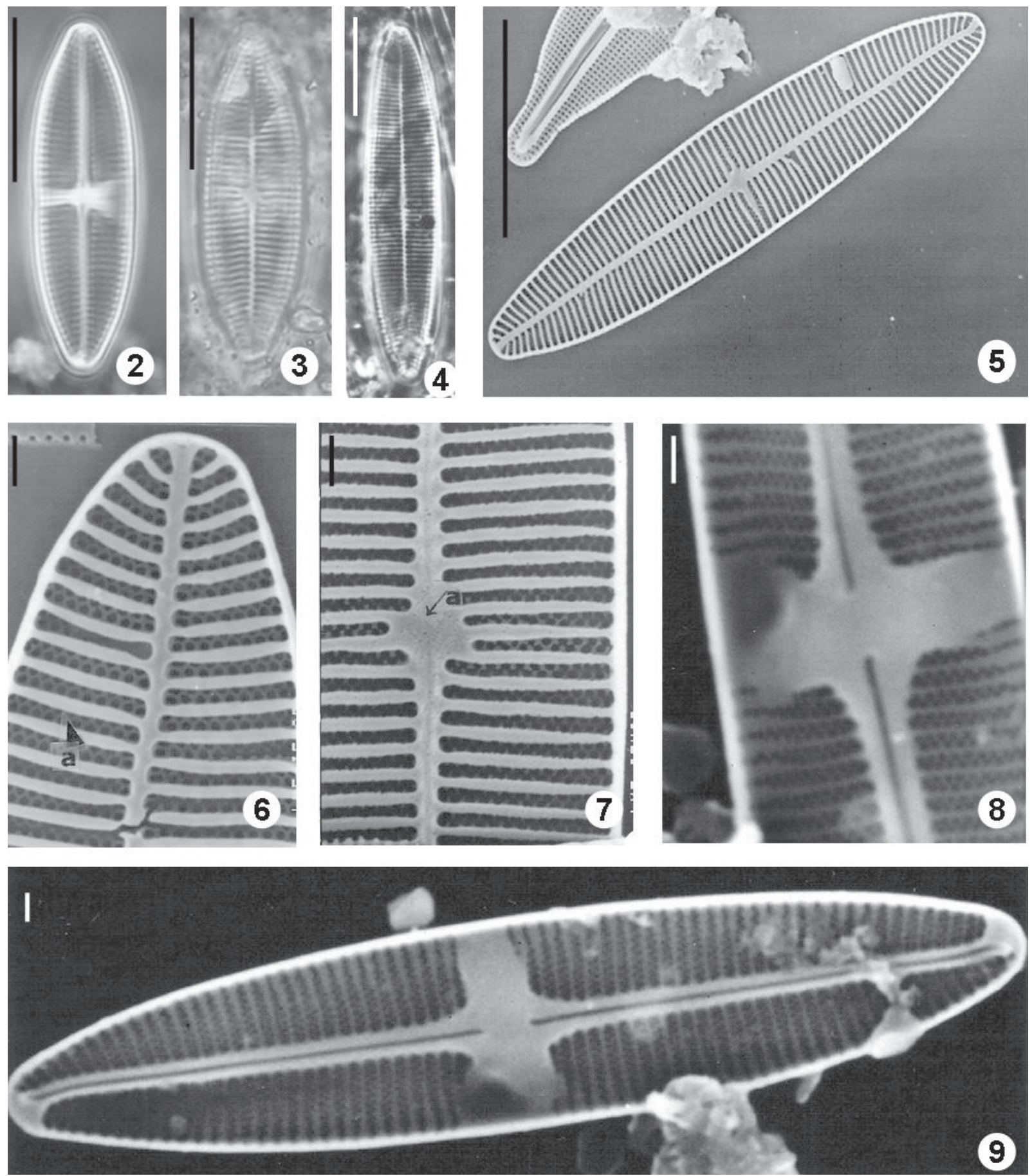

Figuras 2-9. Lemnicola hungarica. Figura 2. Valva rafídea, MO; Figuras 3-4. Valva arrafídea, MO. Figuras 5-7. Valva arrafídea, MEV, Figura 6a (seta): estria bisseriada, Figura 7a (seta): área central circular. Figuras 8-9: Valva rafídea, MEV. Escalas nas figuras 2-5=10 $\mu$ m; $6-9=1 \mu \mathrm{m}$. 
Materiais examinados: BRASIL. São PAULO: São Paulo, Parque Estadual das Fontes do Ipiranga, Lago das Garças, 18-VII-1991, D.C. Bicudo, T.A.V. Ludwig \& D.M. de Figueiredo s.n. (SP255751); 14-I-1997, D.C. Bicudo \& L.L. Morandi (SP294904); Lago das Ninféias, 7-XI-1996, D.C. Bicudo \& L.L. Morandi s.n. (SP294899); Reginópolis, SP-331, km 115,2, à esquerda na direção de Pirajuí, lado direito do Rio Batalha, $500 \mathrm{~m}$ depois da entrada de Reginópolis, 22-II-1992, C.E.M. Bicudo \& D.C. Bicudo s.n. (SP255769); Itapura, Rio Tietê, SP-595, km 21,5, 14-I-1997, D.C. Bicudo \& L.L. Morandi s.n. (SP355388).

De acordo com Patrick \& Reimer (1966) (como Achnanthes hungarica Grun.) e Round \& Basson (1997), Lemnicola hungarica caracteriza-se pela área central da valva rafídea, que é estaurada bilateralmente de modo assimétrico.

As frústulas examinadas apresentaram extremidades cuneadas, sub-rostradas e rostradas, incluindo todas as formas mencionadas para diferentes populações descritas em literatura. O material confere com a bibliografia nacional (Rodrigues 1988, Ludwig \& Flôres 1995, Flôres 1997 e Brassac 1999), e estrangeira (Elmore 1921, Patrick \& Reimer 1966, Compère 1975, Jensen 1985, Krammer \& LangeBertalot 1991, De Dromitrovic \& Maidana 1997), nas quais foi citada como Achnanthes hungarica (Grun.) Grun., bem como com Round \& Basson (1997) e Sala et al. (1999), citada como Lemnicola hungarica (Grun.) Round \& Basson. Ainda, Atab (2000), apesar de identificar corretamente o táxon para dois ambientes lóticos localizados no estado do Paraná, equivocou-se ao caracterizá-lo pela presença de área central unilateralmente expandida na valva sem rafe, pois tal característica é típica da valva com rafe.

A espécie distribuiu-se em $3,1 \%$ das localidades amostradas no Estado de São Paulo, que representaram apenas três municípios (figura 1), indicando ser uma espécie de pequena distribuição no Estado de São Paulo. Ocorreu como parte do perifíton e metafíton de ambientes lóticos e lênticos. Com base em um único registro de dados ambiental (Município de Itapura), foi encontrada em águas com baixa condutividade $\left(40 \mu \mathrm{S} \mathrm{cm}^{-1}\right)$ e $\mathrm{pH}$ levemente ácido $(\mathrm{pH}=6)$. Conforme Germain (1981) e Round \& Basson (1997), a espécie é freqüentemente encontrada aderida a macrófita do gênero Lemna. Apesar desta macrófita não ter sido encontrada no presente estudo, a espécie foi encontrada no hábitat perifítico ou metafítico, ou seja, associada sempre a algum tipo de substrato.

Finalmente, o gênero uniespecífico é citado pioneiramente para o Estado de São Paulo, bem como são inéditas as descrições e ilustrações de características ultra-estruturais (MEV) em publicações brasileiras de cunho efetivo.

\section{Agradecimentos}

À FAPESP, Fundação de Amparo à Pesquisa do Estado de São Paulo, pela bolsa em nível de mestrado concedida à primeira autora (processo n. 00/04230-0) e pelo auxílio concedido ao projeto temático "Flora Ficológica do Estado de São Paulo" (processo 98/04955-3).

\section{Literatura citada}

Aguiar, L. \& Martau, L. 1979. Diatomáceas de lagos do Parque Zoológico, Rio Grande do Sul, Brasil. Iheringia, série Botânica 25: 27-110.

Anonymous. 1975. Proposals for a standartization of diatom terminology and diagnosis. Nova Hedwigia 53: 223-354.

Atab, D. 2000. Diatomáceas (Bacillariophyta) de rios das bacias do Tibagi e do Itararé, centro-oeste do estado do Paraná, Brasil. Dissertação de Mestrado, Universidade Federal do Paraná, Curitiba.

Barber, H.G. \& Harworth, E.Y. 1981. A guide to the morphology of the diatom frustule. Freshwater Biological Association Scientific Publication, $\mathrm{n}^{\circ}$ 44, $113 \mathrm{p}$.

Bigunas, P.I.T. 2005. Diatomáceas (Ochrophyta) do rio Guaraguaçú, litoral do Paraná, Brasil. Dissertação de Mestrado, Universidade Federal do Paraná, Curitiba.

Brassac, N.M. 1999. Diatomoflórula dos rios da área de influência da Usina Hidrelétrica de Salto Caxias Bacia do Rio Iguaçu-Paraná. Dissertação de Mestrado, Universidade Federal do Paraná, Curitiba.

Bukhtiyarova, L. \& Round, F.E. 1996. Revision of the genus Achnanthes sensu lato Psammothidium, a new genus based on A. marginulatum. Diatom Research 11: 1-30.

Carneiro, L.A. 2003. Ordem Achnanthales (Bacillariophyceae) de águas doces do estado de São Paulo: levantamento florístico. Dissertação de Mestrado, Universidade Estadual Paulista, Rio Claro.

Compère, P. 1975. Algues de la région du lac Tchad, 4: Diatomophycées. Hydrobiologie 9: 203-290.

Contin, L.F. 1983. Contribuição ao estudo das diatomáceas (Chrysophyta, Bacillariophyceae) na região da barragem de captação d'água do rio Iguaçu (SANEPAR), em Curitiba, estado do Paraná, Brasil. Dissertação de Mestrado, Setor de Ciências Biológicas, Universidade Federal do Paraná, Curitiba. 
Contin, L.F. 1990. Contribuição ao estudo das diatomáceas (Chrysophyta, Bacillariophyceae) na região da barragem de captação d'água do rio Iguaçu (SANEPAR), em Curitiba, Estado do Paraná, Brasil. Estudos de Biologia 24: 5-95.

Costa, J.C.F. 1995. Diatomáceas (Bacillariophyceae) da Reserva Biológica de Poço das Antas, município de Silva Jardim, Rio de Janeiro, Brasil. Iheringia, série Botânica 46: 57-143.

De Domitrovic, Y.Z. \& Maidana, N.I. 1997. Taxonomic and ecological studies of the Paraná River diatom flora (Argentina). Bibliotheca Diatomologica. v. 34. J. Cramer, Berlin Stuttgart.

Diaz-Villanueva, V. \& Maidana, N.I. 1999. Diatoms (Bacillariophyceae) from Pulmarí Lake (Neuquén, Argentina). Biologia Bratislava 54:11-10.

Elmore, C.J. 1921.The diatoms (Bacillarioideae) of Nebraska. University Studies 21: 22-214.

Ferrari, F. 2004. Diatomáceas dos rios Ivaí, São João e dos Patos, Bacia do Ivaí, município de Prudentópolis, Paraná. Dissertação de Mestrado, Setor de Ciências Biológicas, Universidade Federal do Paraná, Curitiba.

Flôres, T.L. 1997. Inventário florístico das diatomáceas (Bacillariophyta) do Banhado do Taim, Rio Grande do Sul, Brasil. Dissertação de Mestrado, Universidade Federal do Paraná, Curitiba.

Frenguelli, J. 1942. Diatomeas Del Néuquen. Revista Del Museo de La Plata Seccion Botanica 5: 73-219.

Germain, H. 1981. Flore des diatomées. Soucieté Nouvelle des Ëditions Boubée, Paris.

Hasle, G.R. \& Fryxell, G.A. 1970. Diatoms: cleaning and mounting for light and electron microscopy. Transactions of the American Microscopical Society 89: 496-474.

Herbst, N. \& Maidana, N.I. 1989. Diatoms of Chaco (Republica Argentina): 1. Nova Hedwigia 49: 207-232.

Jensen, N.G. 1985. Hustedt's “Die kieselalgen, 2. Teil": The pennate diatoms. Koeltz Scientific Books, Koenigstein.

Krammer, K. \& Lange-Bertalot, H. 1991. Bacillariophyceae 3: Achnanthaceae. In: H. Ettl., J. Gerloff, H. Heynig \& D. Mollenhauer (eds). Süsswasser Flora von Mittleleuropa. Band 2/4. Gustav Fischer Verlag, Stuttgart.

Landucci, M. 2002. Diatomáceas de rios da bacia hidrográfica litorânea, Paraná, Brasil: Coscinodiscophyceae, Fragilariophyceae e Bacillariophyceae (Achnanthales e Eunotiales). Dissertação de Mestrado, Setor de Ciências Biológicas, Universidade Federal do Paraná, Curitiba.

Leandrini, J.A. 1999. Diatomáceas perifíticas da região de captação de água da SANEPAR,Maringá - Paraná (córrego Sarandi e rio Pirapó). Dissertação de Mestrado, Setor de Ciências Biológicas, Universidade Federal do Paraná, Curitiba.
Ludwig, T.A.V. \& Flôres, T.L. 1995. Diatoms of the rivers from the region to be dammed for the construction of Segredo Hydroelectric, Paraná, Brazil. Arquivos Biologia e Tecnologia 38: 631-650.

Maidana, N.I. \& Diaz-Villanueva, V. 2001. Diatomeas de Lagos Oligotroficos Andinos, (Provincia de Neuquén Argentina). Boletin Sociedad Argentina Botanica 36: 15-27.

Maidana, N.I. \& Herbst, N. 1994. Diatomeas Del Chaco (Argentina): II. Boletin Sociedad Argentina Botanica 30: 25-42.

Maidana, N.I., Vigna, M.S. \& Mascitti, V. 1998. Ficoflora de La Laguna de Pozuelos (Jujuy, Argentina) I: Bacillariophyceae. Boletin Sociedad Argentina Botanica 33: 171-179.

Moreira Filho, H. \& Valente-Moreira, I. 1981. Avaliação taxonômica e ecológica das diatomáceas (Bacillariophyceae) epífitas em algas pluricelulares obtidas nos litorais do Paraná, Santa Catarina e São Paulo. Boletim do Museu Botânico Municipal de Curitiba. 47: 1-17.

Patrick, R. \& Reimer, C.W. 1966. The diatoms of United States. Academy of Natural Sciences, Philadelphia.

Rodrigues, L. 1984. Contribuição ao conhecimento das diatomáceas do Rio Tubarão, Santa Catarina, Brasil. Insula 14: 47-120.

Rodrigues, L. 1988. Diatomoflorúla da região de captação de água do Rio Tubarão, município de Tubarão, Santa Catarina. Dissertação de mestrado, Universidade Federal do Paraná, Curitiba.

Rodrigues, L. \& Moreira-Filho, H. 1990. Diatomoflórula do Rio Tubarão, Santa Catarina, Brasil: I- Eunotiaceae Kützing e II- Achnanthaceae. Insula 20: 113-136.

Rosa, Z., Ungaretti, I. \& Kremer, L. 1987. Ficoflórula de ambientes lênticos - estudo preliminar da região de Charqueadas, Rio Grande do Sul, Brasil, com vistas à avaliação ambiental. Acta Botanica Brasilica 1: 165-188.

Ross, R., Cox, E.J., Karayeva, N., Mann, D.G., Padock, T.B.B., Simonsen, R. \& Sims, P.A. 1979. An amended terminology for the siliceous components of the diatom cell. Beihefte zur Nova Hedwigia 64: 553-513.

Round, F.E. \& Basson, P.W. 1997. Anew monoraphid diatom genus (Pogoneis) from bahrain and the transfer of previously described species $A$. hungarica and $A$. taeniata to new genera. Diatom Research 12: 71-81.

Round, F.E. \& Bukhtiyarova, L. 1996a. Four new genera based on Achnanthes (Achnanthidium) together with a re-definition of Achnanthidium. Diatom Research 11: 345-361.

Round, F.E. \& Bukhtiyarova, L. 1996b. Epipsamic diatoms Communities of British Rivers. Diatom Research 11:363-372.

Round, F.E., Crawford, R.M. \& Mann, D.G. 1990. The diatoms: biology, morphology of the genera. Cambridge University Press, Cambridge. 
Sala, S.E., Santiago, R.D., Nuñes-Avellaneda \& Lamaro,A.A. 1999. Nuevos registros de diatomeas (Bacillariophyceae) de la amazônia colombiana. Caldasia 21:26-37.

Shirata, M.T. 1986. Contribuição ao estudo das diatomáceas (Bacillariophyceae) no lago do Parque São Lourenço, Curitiba, estado do Paraná, Brasil. Dissertação de Mestrado, Setor de Ciências Biológicas, Universidade Federal do Paraná, Curitiba.

Simonsen, R. 1974. The diatom plankton of the Indian ocean expedition of R/V "Meteor", 1964-1965. "Meteor" Forschungsergebnisse, Reihe D 19:1-107.

Torgan, L., Becker,V. \& Prates, H.M. 1999. Checklist das diatomáceas (Bacillariophyta) de ambientes de águas continentais e costeiras do Rio Grande do Sul, Brasil. Iheringia, série Botânica 52: 89-144.
Valente-Moreira, I.M, Moreira Filho, H. \& Cunha, J. A. 1994. Diatomáceas (Chrysophyta, Bacillariophyceae) em biótopo do manguezal do rio Perequê, em Pontal do Sul, Paranaguá, Estado do Paraná, Estado do Paraná, Brasil. Acta Biológica Paranaense 23: 55-72.

Visinoni, N.D. 2002. Diatomáceas de rios da região metropolitana de Curitiba, Paraná, Brasil: Coscinodiscophyceae, Fragilariophyceae e Bacillariophyceae (Achnanthales e Bacillariales). Dissertação de Mestrado, Setor de Ciências Biológicas, Universidade Federal do Paraná, Curitiba.

West, B.S. 1914. A contribution to our knowledge of freshwater algae of Colombia. In: O. Furhumann, \& E. Mayor (eds.). Voyage d'exploration scientifique in Colombie. Attinger, Neuchâtel, pp. 1013-1051. 